\title{
Brachytherapy for malignancies of the vagina in the 3D era
}

\author{
Scott M. Glaser, MD, Sushil Beriwal, MD \\ Department of Radiation Oncology, University of Pittsburgh Cancer Institute, Pittsburgh, PA, USA
}

\begin{abstract}
Vaginal cancer is an uncommon malignancy and can be either recurrent or primary. In both cases, brachytherapy places a central role in the overall treatment course. Recent technological advances have led to more advanced brachytherapy techniques, which in turn have translated to improved outcomes for patients with malignancies of the vagina. The aim of this manuscript is to outline the incorporation of modern brachytherapy into the treatment of patients with vaginal cancer including patient selection along with the role of brachytherapy in conjunction with other treatment modalities, various brachytherapy techniques, treatment planning, dose fractionation schedules, and normal tissue tolerance.
\end{abstract}

Key words: brachytherapy, intracavitary, interstitial, vaginal cancer.

\section{Purpose}

Vaginal cancer, whether recurrent or primary, is a rare malignancy. There are 3,000-4,000 cases of primary vaginal cancer diagnosed each year in the United States $[1,2]$. As an uncommon disease, there are no published National Comprehensive Cancer Network (NCCN) guidelines. Radiotherapy, particularly brachytherapy, is fundamental in the treatment of both primary and recurrent vaginal cancers [3-8]. The complete treatment regimen for patients with vaginal cancer usually consists of brachytherapy (BT) in conjunction with external beam radiation therapy (EBRT), though in some instances can be either alone [9-11]. Recent national trends in utilization have shown an alarming decrease in BT boost in favor of other modalities, despite extensive published literature in support of BT, and relative scarceness of data supporting other modalities [12]. In this review, we aim to give an overview of the incorporation of modern BT into the treatment of vaginal cancer.

\section{Patient selection}

\section{External beam radiation}

The definitive management of both primary and recurrent vaginal cancer should include a course of EBRT in order to address potential micrometastatic nodal disease and to downsize the primary tumor prior to BT [13]. Some patients may be at low risk for nodal disease and could potentially be cured with BT alone (especially those with small, superficial disease), though this cohort remains difficult to distinguish and has not been prospectively defined. Typically, 45-50.4 Gy is delivered in 25-28 fractions to the primary site including the entire vagina, the paravaginal area to the pelvic sidewalls, and the gross tumor volume with margin, as well as to the lymph nodes at risk including the distal common iliac, internal iliac, external iliac, obturator, presacral, and in cases of involvement of the distal third to half of the vagina, the inguinal nodes.

\section{Chemotherapy}

The role of systemic therapy in the setting of primary vaginal cancer has not been prospectively evaluated. However, a recent analysis of the National Cancer Database demonstrated a trend for increased utilization of concurrent chemotherapy (nearly 60\% in 2011 and 2012), and that concurrent chemotherapy was associated with an absolute 5-year survival benefit of $6.9 \%$ [14]. The survival benefit was seen in all stages, though to the greatest degree in stages II-IV. Extrapolating from the cervical cancer experience, chemotherapy is typically platinum-based $[15,16]$. The role of systemic therapy in the setting of recurrent tumors of the vagina is less clear, though this is the subject an ongoing study, GOG 238, which will hopefully help answer this question. Our institutional practice is to offer concurrent cisplatin for patients with bulky or high grade recurrences and for all patients with nodal recurrence.
Address for correspondence: Sushil Beriwal, MD, Department of Radiation Oncology, University of Pittsburgh Cancer Institute, Magee Womens Hospital of UPMC (Radiation-Oncology), 300 Halket St, Pittsburgh PA 15213, USA, phone: +1 412-641-4600, fax: +1 412-641-6601, ® e-mail: beriwals@upmc.edu
Received: 13.08 .2015

Accepted: 23.08 .2015

Published: 30.08 .2015 


\section{Brachytherapy}

Brachytherapy is indicated in nearly all patients with primary or recurrent vaginal cancer. The primary exception is patients who are medically unable to tolerate the BT procedure including any associated anesthesia. In order to deliver adequate dose to the target with high conformality and to limit dose to critical organs, numerous BT techniques have been developed. These include single-channel vaginal cylinder brachytherapy (SCVCB), multi-channel vaginal cylinder brachytherapy (MCVCB), and interstitial BT. The decision of which brachytherapy technique to employ is influenced by multiple factors, with tumor location, extent of disease, and response to EBRT as the main factors. Tumors can regress significantly with EBRT, making it important to evaluate extent of disease before and after EBRT. Modern three-dimensional conformal BT techniques have allowed for a growing emphasis on maintaining acceptable dosages to organs at risk (OAR).

\section{Imaging}

In order to plan for BT, cross-sectional imaging is crucial. We strongly advocate for the use of Magnetic Resonance Imaging (MRI) whenever available, with both a pre-EBRT and post-EBRT diagnostic MRI. At our institution, we perform an MRI with vaginal gel for optimal distention of the vagina, which helps better visualize mucosal extent of disease (Figure 1).

\section{Brachytherapy techniques}

\section{Single channel vaginal cylinder}

The simplest form of vaginal BT is with a single-channel cylinder. This was the first type of intracavitary applicator to be widely adapted into the treatment of vaginal cancer, and historically was the most frequently used.

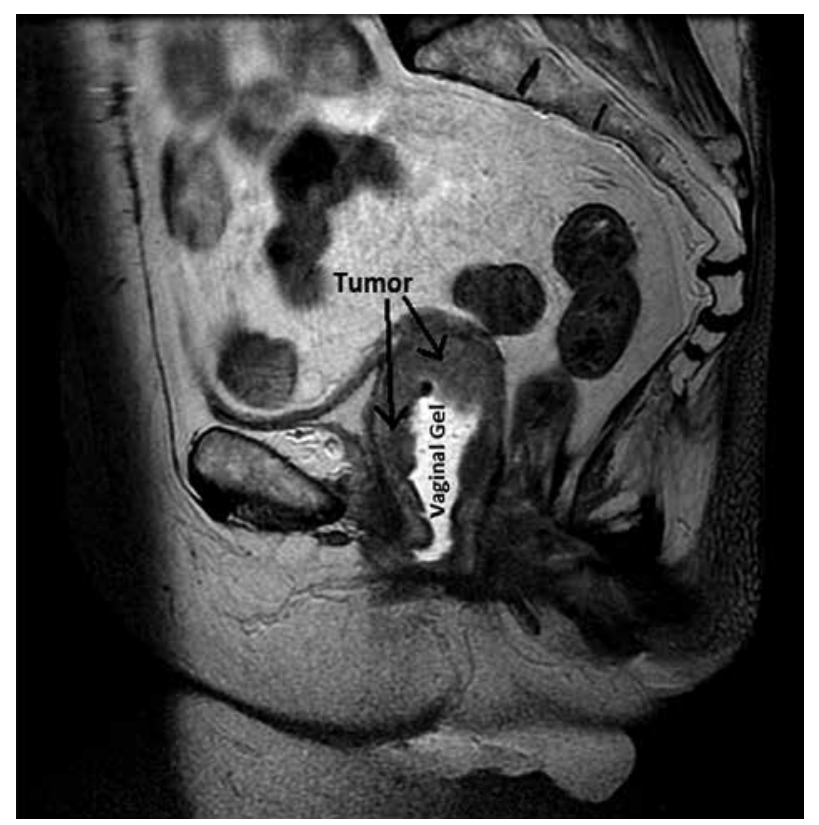

Fig. 1. Sagittal view T2 sequence MRI with vaginal gel, demonstrating the extent of tumor
The basic nature of SCVCB allow the dosimetric properties to be easily understood; however, at the same time limits the utility in the modern treatment of vaginal malignancies. Due to the single central channel, it is not possible to preferentially deliver dose to certain walls of the vagina while avoiding others, though by adjusting dwell times, plans can be created to dose distal and proximal portions of the vagina differently. The dose delivered to tissue falls off according to the inverse square law. Thus, when prescribing dose to a certain depth from the vaginal surface, tissue between the prescription point and the central source receives an exponentially higher dose the closer to the central source it is. This has important implications concerning cylinder size [17].

Commercially available vaginal cylinders range in diameter from $2 \mathrm{~cm}$ to $4 \mathrm{~cm}$. When a smaller cylinder is used, the ratio of prescription depth to cylinder radius is greater. Following the inverse square law, this creates higher surface doses for smaller cylinders. For example, if a $2.5 \mathrm{~cm}$ cylinder were used with a prescription depth of $5 \mathrm{~mm}$, the dose to the vaginal surface (tissue-cylinder interface) would be $196 \%$ of the prescription dose, whereas if a $3.5 \mathrm{~cm}$ cylinder were used with the same prescription depth of $5 \mathrm{~mm}$, the vaginal surface dose would be reduced to $165 \%$ of the prescription dose. As such, when performing intracavitary BT, the largest cylinder, which the patient can comfortably accommodate should be used. This also helps prevent air gaps between the tissue and the cylinder, which are dosimetrically disadvantageous. An example of a SCVCB plan can be found in Figure 2.

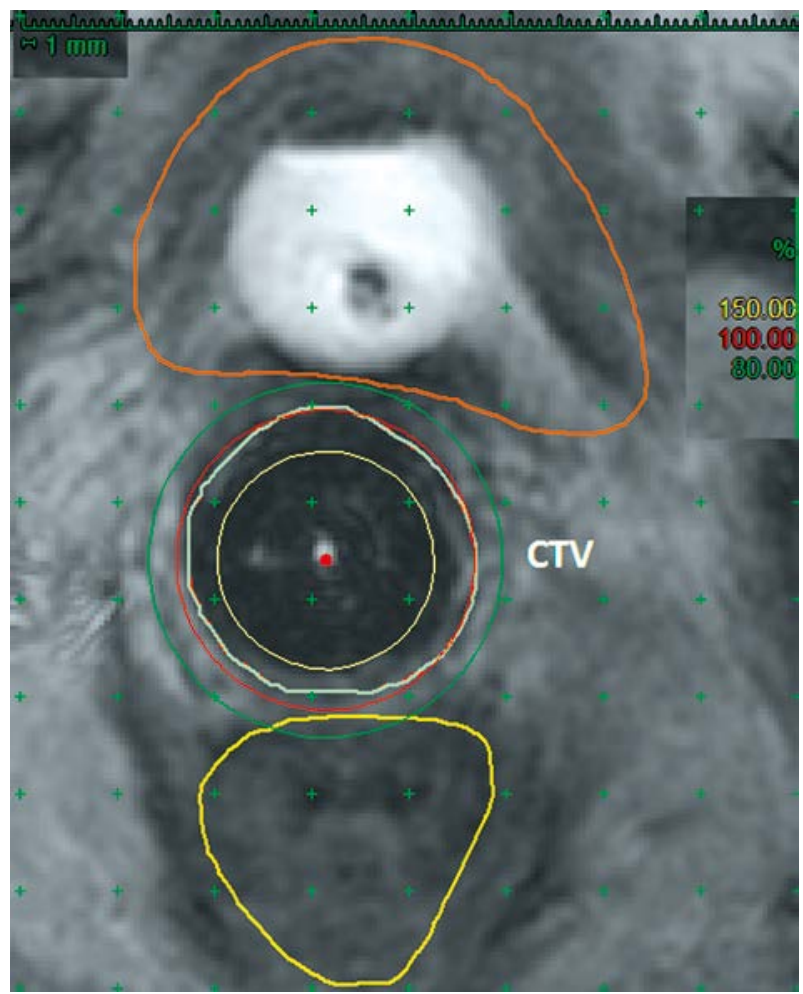

Fig. 2. Dosimetry of a single-channel vaginal cylinder with prescription dose of $45 \mathrm{~Gy}$ in 25 fractions of EBRT followed by $5 \mathrm{~Gy} \times 5$ fractions HDR brachytherapy with CTV $\mathrm{D}_{90}=75.5 \mathrm{~Gy}$, Rectum $\mathrm{D}_{2 \mathrm{cc}}=61.6 \mathrm{~Gy}$, Bladder $\mathrm{D}_{2 \mathrm{cc}}=58.6 \mathrm{~Gy}$ 
Due to the inability to sculpt dose with a single central channel and concerns for associated dose to nearby OAR, SCVCB utilization in the modern era is primarily limited to instances where the entire circumference of the vagina is desired to receive the same dose, such as for adjuvant treatment of the vaginal cuff after hysterectomy for endometrial cancer or apical recurrences with residual disease thickness $\leq 5 \mathrm{~mm}$ [18]. In situations where dose to a particular region must be restricted, SCVCB with shielding can be employed. At our institution, SCVCB is not routinely used in the setting of vaginal cancer.

\section{Interstitial brachytherapy}

By placing needles directly in and around the tumor, numerous source dwell positions can be used and the source can be in closer proximity to the target. This allows for substantially better shaping of dose to fit an individual target. However, there is cost associated with this increased conformality as interstitial brachytherapy typically requires violation of tissue places, anesthesia, prolonged immobilization, and hospitalization, and therefore has a higher likelihood of procedural morbidity than intracavitary BT.

The American Brachytherapy Society (ABS) guidelines recommend interstitial BT to be used for lesions greater than $5 \mathrm{~mm}$ in thickness after EBRT [19]. In order to achieve the best outcomes, a multidisciplinary approach involving gynecological oncology, radiation oncology, and radiology is recommended. Generally, patients should be taken to the operating room and placed under general anesthesia. An exam under anesthesia should be performed and information collected from this exam, as well as MRI of the pelvis pre- and post-EBRT should be used to plan needle placement. Gold fiducial markers can be placed at the proximal and distal end of residual disease, so that it can be identified on computed tomography (CT) scan for defining target volume. Generally, a perineal template should be used for needle placement, though for distal lesions one can place free hand needles under palpation and then anchor the needles to the perineum. Commercially available needles contain catheters made of titanium, stainless steel, or plastic. Plastic catheters have the advantage of being MRI compatible, though are not as rigid and may be more prone to splaying. Our institutional preference is to use titanium catheters. Needles should be placed such that they extend approximately 5-10 mm beyond the tumor, provided this can be accomplished without perforating critical structures. Consideration for laparoscopic guidance is recommended in the setting of apically located lesions, especially if there is supravaginal extension or close proximity of small bowel loop or sigmoid to the tumor. Needles should be placed in a pattern such that there is approximately $10 \mathrm{~mm}$ between each and with attention to location of OAR to avoid perforation. Epidural anesthesia is utilized to control pain while the implant remains in place. A post-implant cross-sectional scan should be taken for dosimetric planning and needles adjusted under epidural for optimization of placement (CT scan should be utilized if stainless steel or titanium needles, as titanium is only compatible with MRI up to 0.3 tesla magnet, and MRI should be utilized with plastic needles). An example of an interstitial plan can be found in Figure 3. Even with sutured fixation of the template, interfraction variation in applicator placement is possible, and if displacement is suspected, repeat imaging should be performed with adjustment of catheters and/or dosimetry $[20,21]$.

\section{Multichannel vaginal cylinder}

In order to try and bridge the gap between the tolerability of SCVCB and the dosimetric advantages of interstitial brachytherapy, more modern brachytherapy applicators have been developed, with the most prominent being MCVCB. A typical multichannel vaginal cylinder has one central channel and 6-8 peripheral channels organized circumferentially around the central channel approximately 5 to $7 \mathrm{~mm}$ from the cylinder surface. These cylinders come in various sizes, with the most common being 3 and $3.5 \mathrm{~cm}$ in diameter. By utilizing multiple source positions in closer proximity to the target, MCVCB allows dose to be preferentially delivered to specific portions of the vagina. This translates into considerable advantages as compared to SCVCB as the target is able to be more adequately covered while simultaneously decreasing dose to OAR [22-24]. An example of a MCVCB plan can be found in Figure 4B. Still, there are limitations as to
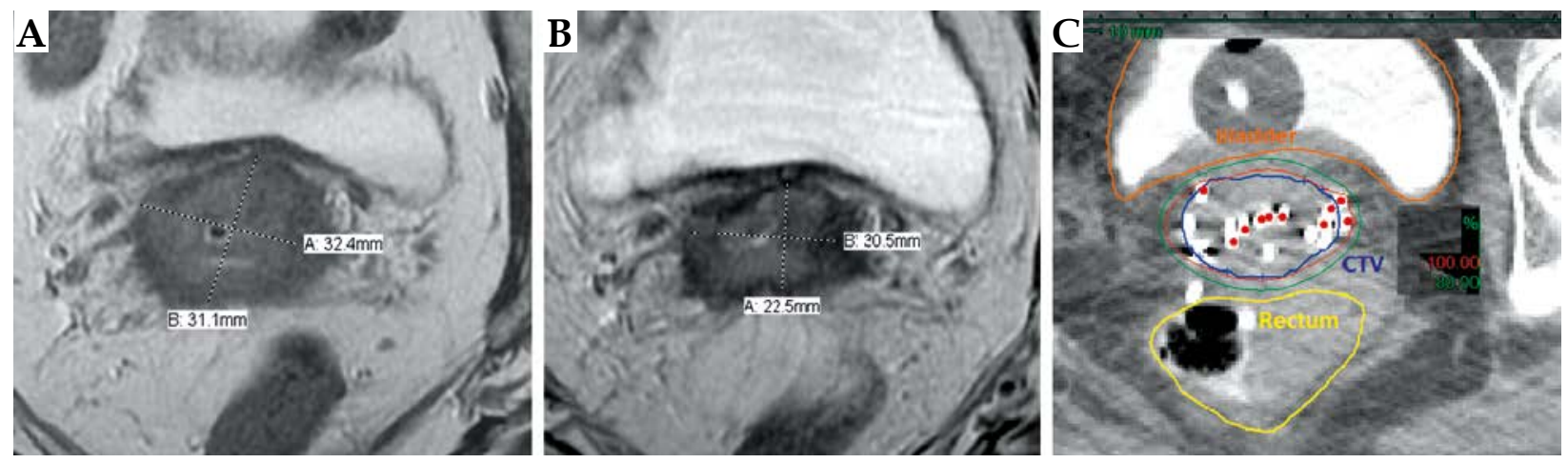

Fig. 3. Dosimetry of an interstitial brachytherapy implant in a patient with adenocarcinoma and a poor response to EBRT with prescription dose of $45 \mathrm{~Gy}$ in 25 fractions of EBRT followed by $5.5 \mathrm{~Gy}$ x 5 HDR brachytherapy. A) Pre-EBRT MRI. B) Post-EBRT MRI. C) Interstitial Plan: $C T V D_{90}=82.1 \mathrm{~Gy}$, Rectum $\mathrm{D}_{2 \mathrm{cc}}=60.0 \mathrm{~Gy}$, Bladder $\mathrm{D}_{2 \mathrm{cc}}=58.6 \mathrm{~Gy}$ 

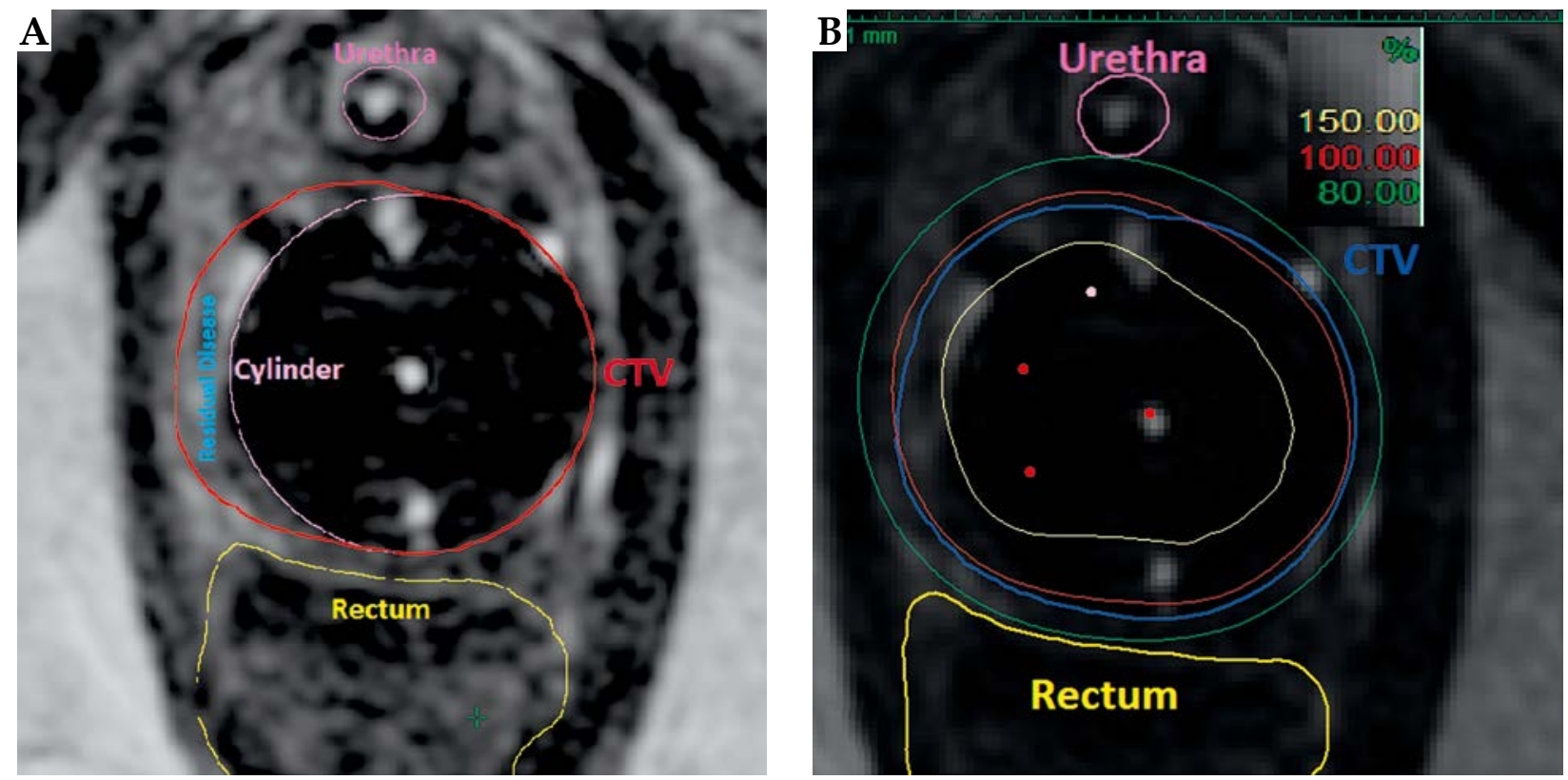

Fig. 4. Dosimetry of a multi-channel vaginal cylinder. A) Contouring. B) Plan with prescription dose of 45 Gy in 25 fractions EBRT and 5.5 Gy x 5 fractions HDR brachytherapy with CTV $\mathrm{D}_{90}=79.8 \mathrm{~Gy}$, Rectum $\mathrm{D}_{2 \mathrm{cc}}=59.8 \mathrm{~Gy}$, Bladder $\mathrm{D}_{2 \mathrm{cc}}=54.1 \mathrm{~Gy}$, Urethra $\mathrm{D}_{0.1 \mathrm{cc}}=71.3 \mathrm{~Gy}$

the size of lesions, which can be treated with MCVCB. As the thickness of the lesion increases so does the dose to the vaginal mucosa, and for lesions located away from the rectum, vaginal mucosal dose is typically the dose limiting structure. At our institution, we use a tumor thickness cut-off of approximately $5 \mathrm{~mm}$ for apically or posteriorly located lesions and $7 \mathrm{~mm}$ for other locations, provided dose constraints to other OAR are met. We recently published on a series of 41 patients, all of whom were treated with image-based high-dose-rate (HDR) MCVCB, and demonstrated excellent local-control and low toxicities, with an overall favorable comparison to historical results with SCVCB [25].

\section{Fractionation schedules}

When discussing doses prescribed with vaginal brachytherapy, it is important to note the effects of dose per fraction and dose rate. One widely accepted way to standardize the dose prescribed is to calculate the biologically equivalent dose had the treatment been delivered with standard fractionation at 2 Gy per day (EQD2). An excellent resource for doing so is given by Nag et al. including instructions for a spreadsheet based dose calculator [26]. The ABS also provides a spreadsheet based EQD2 calculator (https://www.americanbrachytherapy.org/guidelines/LQ_spreadsheet.xls). Historically, brachytherapy was delivered primarily with low-dose-rate (LDR) isotopes, however more recently HDR via remote afterloading has been the predominant modality [27-29]. The physics of afterloading HDR vaginal cylinder have been well described [30]. The advantages of HDR include the ability to customize dose after the implant by adjusting dwell times and weighting, shorter treatment time, which allows for more outpatient treat- ment, better documentation, and a decreased exposure to radiation for health care workers [31].

There has not been a prospective trial comparing brachytherapy fractionation schedules and recommendations on dose are primarily based on single-institution reports and consensus guidelines. The typical prescription dose for vaginal malignancies is 70-80 Gy EQD2 including the contribution from the combination of EBRT and BT. Various fractionation schedules exist to deliver a dose in this range. At our institution, brachytherapy is predominately delivered in 5 fractions with a dose of 4.55.5 Gy per fraction after 45 Gy in 25 fractions of EBRT (EQD2 of 71.4-79.8 Gy). Another commonly used schedule is $3 \mathrm{~Gy} \times 9-10$ fractions (EQD2 of 73.5-76.8 Gy). The final decision on what dose to prescribe is dependent upon tumor location, response to EBRT, extent of residual disease, and critical organ dosimetry. Dose escalation up to 85 Gy may be appropriate for bulky tumors and/or those with poor response to EBRT as long as normal tissue dose constraints are respected.

\section{Treatment planning}

Details of brachytherapy planning are beyond the scope of this review; however, collaboration with a physicist who has experience with gynecological brachytherapy is essential. In order to accurately calculate dose to OAR, it is imperative that both the target and OAR be contoured appropriately. Whenever possible, we recommend MRI imaging to be incorporated into treatment planning either by getting a pre-brachytherapy MRI scan or MRI scan with applicator in situ. Detailed recommendations for contouring have been given by both GECESTRO, and the ABS [19,32]. At our institution, we define clinical target volume (CTV) for dose prescription 
based on both pre and post EBRT imaging and clinical examination. We treat pre-EBRT involved length of vagina and include the entire circumference of vagina for that length. For the involved wall of the vagina, we include the entire thickness of vaginal wall plus residual disease seen on imaging, while for the uninvolved vaginal walls we contour only up to the mucosal surface (Figure 4A). Of note, it is critical to precisely contour the portion of the OAR in closest proximity to needles or applicator as this region is likely to be the dose-limiting region, and accuracy in determining absolute dose here will effectively help reduce the risk of side effects. At our institution, we aim for $90 \%$ of the target volume to be covered by at least $100 \%$ of the prescription dose $\left(D_{90} \geq 100 \%\right)$. We try to limit hot spots by keeping the CTV volume to receive $150 \%$ of the prescription dose $\left(\mathrm{V}_{150}\right)$ to less than $50 \%$ and the $\mathrm{V}_{200}<15-20 \%$. We employ manual optimization for intracavitary BT, and a combination of manual and graphical optimization for interstitial BT.

If an odd number of fractions of interstitial BT are to be delivered, it is in the patient's best interest to attempt to place the implant in the morning, and have the first fraction planned and delivered by that afternoon in order to minimize the period of immobilization and length of hospital stay.

\section{Tolerance of organs at risk}

The primary toxicities associated with brachytherapy for vaginal cancer are related to the rectum, bladder, urethra, vaginal mucosa, and bowel. The tolerance of the rectum and bladder has been well defined with general agreement among single institution experiences and consensus guidelines, with recommended EQD2 to the most irradiated $2 \mathrm{cc}\left(\mathrm{D}_{2 \mathrm{cc}}\right)$ for the bladder of less than $90 \mathrm{~Gy}$ and less than 70-75 Gy for the rectum [33-35]. These sources suggest that if these constraints are met, the rates of grade 2 or higher toxicities can be limited to less than $10 \%$. The tolerance to the vaginal mucosa is not as well defined. To this point, ABS consensus guidelines avoid giving a precise constraint $[19,35,36]$. The upper portion of the vagina is thought to be more tolerant to radiation than the lower portion, likely due to the thinner rectovaginal septum and closer proximity to the urethra in the lower vagina [37]. In the modern, image-based era, a Japanese group found vaginal EQD2 $\mathrm{D}_{2 \mathrm{cc}}$ of $145 \mathrm{~Gy}$ or

Table 1. Summary of local control and late toxicities for vaginal cancer patients treated with brachytherapy

\begin{tabular}{|c|c|c|c|c|c|c|c|}
\hline Primary technique & $\begin{array}{c}\text { First author } \\
\text { (year of publication) }\end{array}$ & $n$ & $\begin{array}{c}\% \text { recurrent/ } \\
\% \text { primary }\end{array}$ & Local control & $\begin{array}{l}\text { Vaginal } \\
\text { toxicity }\end{array}$ & $\begin{array}{l}\text { Urinary } \\
\text { toxicity }\end{array}$ & GI toxicity \\
\hline$E B R T+2 D L D R$ & Curran (1988) [42] & 47 & $100 / 0$ & $42 \% 5-y r$ & $0 \%$ & $0 \%$ & $4 \%$ \\
\hline $\mathrm{EBRT}+2 \mathrm{D}$ LDR & Stock (1992) [43] & 49 & $0 / 100$ & $44 \% 2-y r$ & $8 \%$ & $4 \%$ & $14 \%$ \\
\hline $\mathrm{EBRT}+2 \mathrm{D}$ LDR & Morgan (1993) [44] & 34 & $100 / 0$ & $85 \% 5-y r$ & $12 \%$ & $0 \%$ & $0 \%$ \\
\hline $\mathrm{EBRT}+2 \mathrm{D}$ LDR & Sears (1994) [45] & 45 & $100 / 0$ & $54 \% 5-y r$ & $0 \%$ & $4 \%$ & $16 \%$ \\
\hline $\mathrm{EBRT}+2 \mathrm{D}$ LDR & Chyle (1996) [46] & 301 & $0 / 100$ & $77 \% 5-y r$ & $6 \%$ & $4 \%$ & $6 \%$ \\
\hline $\mathrm{EBRT}+2 \mathrm{D}$ LDR & Fine (1996) [47] & 55 & $0 / 100$ & $78 \% 5-y r$ & $0 \%$ & $0 \%$ & $15 \%$ \\
\hline $\mathrm{EBRT}+2 \mathrm{D}$ LDR & Jereczek (2000) [48] & 73 & $100 / 0$ & $40 \% 5-y r$ & $4 \%$ & $4 \%$ & $15 \%$ \\
\hline $\mathrm{EBRT}+2 \mathrm{D}$ LDR & Wylie (2000) [49] & 58 & $100 / 0$ & $65 \% 5-y r$ & NR & NR & NR \\
\hline $\mathrm{EBRT}+2 \mathrm{D}$ LDR & Jhingran (2003) [9] & 91 & $100 / 0$ & $75 \% 5-y r$ & $0 \%$ & $1 \%$ & $11 \%$ \\
\hline $\mathrm{EBRT}+2 \mathrm{D}$ LDR & $\operatorname{Lin}(2005)$ [50] & 50 & $100 / 0$ & $72 \% 10-y r$ & $0 \%$ & $0 \%$ & $10 \%$ \\
\hline $\mathrm{EBRT}+2 \mathrm{D} \mathrm{HDR}$ & Pai (1997) [51] & 20 & $100 / 0$ & $74 \% 4-y r$ & $5 \%$ & $0 \%$ & $5 \%$ \\
\hline $\mathrm{EBRT}+2 \mathrm{D} \mathrm{HDR}$ & Mock (2003) [10] & 86 & $0 / 100$ & $\begin{array}{c}\text { NR } \\
\text { (66\% 5-yr DSS) }\end{array}$ & $4 \%$ & $1 \%$ & $2 \%$ \\
\hline $\mathrm{EBRT}+2 \mathrm{D} \mathrm{HDR}$ & Lieskovsky (2004) [52] & 54 & $0 / 100$ & $87 \% 4-y r$ & $7 \%$ & $2 \%$ & $6 \%$ \\
\hline$E B R T+2 D H D R$ & Petignat (2006) [4] & 22 & $100 / 0$ & $100 \% 5-y r$ & $50 \%$ & $0 \%$ & $18 \%$ \\
\hline $\mathrm{EBRT}+2 \mathrm{D} \mathrm{HDR}$ & Sorbe (2013) [7] & $25^{\#}$ & $100 / 0$ & $92 \% 5-y r$ & $19 \%$ & $4 \%$ & $35 \%$ \\
\hline EBRT + 3D interstitial HDR & Dimopoulos (2012) [53] & 13 & $0 / 100$ & $92 \% 3-y r$ & $15 \%$ & $8 \%$ & $0 \%$ \\
\hline EBRT + 3D interstitial HDR & Beriwal (2012) [54] & 30 & $43 / 57$ & $79 \% 2-\mathrm{yr}^{\dagger}$ & $7 \%$ & $0 \%$ & $0 \%$ \\
\hline $\mathrm{EBRT}+3 \mathrm{D} \mathrm{HDR}$ & Lee (2013) [55] & $31^{*}$ & $100 / 0$ & $96 \% 2-y r$ & $3 \%$ & $0 \%$ & $0 \%$ \\
\hline IMRT + 3D MCVC HDR & Vargo (2015) [23] & 41 & $76 / 24$ & $93 \% 2-y r$ & $0 \%$ & $0 \%$ & $4 \%$ \\
\hline
\end{tabular}

EBRT-external beam radiotherapy, LDR - low-dose-rate, HDR-high-dose-rate, IMRT-intensity modulated radiotherapy, 2D-2-dimensional, 3D - 3-dimensional, MCVC - multichannel vaginal cylinder, GI - gastrointestinal

*Excludes patients with prior pelvic radiotherapy. ${ }^{*}$ Excludes patients treated with EBRT or brachytherapy alone. ${ }^{\dagger}$ Includes regional failures and patients with prior RT 
higher predicted for increased risk of vaginal ulcer $(23.5 \%$ versus $3.7 \%$ ) [38]. On the contrary, the group from Vienna found no correlation of vaginal $\mathrm{D}_{2 \mathrm{cc}}$ with the incidence of vaginal side effects with mean vaginal EQD2 $\mathrm{D}_{2 \mathrm{cc}}$ of $140 \mathrm{~Gy}$ and mean vaginal EQD2 $\mathrm{D}_{0.1 \mathrm{cc}}$ of $750 \mathrm{~Gy}$ [39]. Both groups used the LENT/SOMA morbidity index, though, the Japanese group looked only at ulcer.

There is limited data to guide recommendations regarding urethral and small bowel dosimetry. A small series of patients with vaginal cancer treated with HDR brachytherapy suggested limiting the $D_{0.1 \mathrm{cc}}$ to $\leq$ prescription dose and avoiding any hot spot in the urethra, as those who had severe urethral toxicity had a mean equivalent dose to the urethra of $95 \mathrm{~Gy}$ versus $73 \mathrm{~Gy}$ for those without severe toxicity [40]. Dose to small bowel is difficult to quantify due to interfraction variability in position, and there is limited outcomes data relating to toxicity with gynecological brachytherapy, though, recommendations for increased reporting have been made [41]. At our institution, we attempt to limit small bowel dose by attempting to move bowel in close proximity to the target out of the way with bladder filling, keeping any hot spots out of the bowel, and limiting the EQD2 maximal point dose to $60-65 \mathrm{~Gy}$.

\section{Discussion and conclusions}

Incorporation of modern 3-dimensional imaging modalities into the brachytherapy regimen has resulted in a momentous step forward for vaginal cancer patients. Outcomes data suggest that image-based brachytherapy increases the therapeutic ratio by both increasing local control and decreasing morbidity (Table 1). Due to patient overlap with our interstitial and MCVCB series, we excluded our image-based brachytherapy salvage series from the table, though were able to demonstrate over $90 \%$ local control and low side effects in the purely salvage setting using a combination of interstitial and intracavitary techniques [8]. In the modern era, the use of 3D imaging is paramount due to the close proximity of critical organs, which is under assessed with $2 \mathrm{D}$ planning. The type of brachytherapy to use is a matter of tumor location, extent, and response to EBRT as well as the expertise available. However, in general, patients with proximally located tumors less than $5 \mathrm{~mm}$ in thickness, post-EBRT should be considered for MCVCB, while those with more extensive lesions or lesions in close proximity to OAR or the distal vagina should be considered for interstitial brachytherapy.

\section{Disclosure}

Authors report no conflict of interest.

\section{References}

1. Siegel R, Ma J, Zou Z et al. A. Cancer Statistics, 2014. CA Cancer J Clin 2014; 64: 9-29.

2. Siegel R, Miller K, Jemal. A. Cancer Statistics, 2015. CA Cancer J Clin 2015; 65: 5-29.

3. Nag S, Yacoub S, Copeland LJ et al. Interstitial brachytherapy for salvage treatment of vaginal recurrences in previously unirradiated endometrial cancer patients. Int J Radiat Oncol Biol Phys 2002; 54: 1153-1159.

4. Petignat $\mathrm{P}$, Jolicoeur M, Alobaid A et al. Salvage treatment with high-dose rate brachytherapy for isolated vaginal endometrial cancer recurrence. Gynecol Oncol 2006; 101: 445-449.

5. Beriwal S, Bhatnagar A, Heron DE et al. High-dose-rate interstitial brachytherapy for gynecologic malignancies. Brachytherapy 2006; 5: 218-222.

6. BeriwalS, Heron D, Mogus R et al. High-dose rate brachytherapy (HDRB) for primary or recurrent cancer in the vagina. Radiat Oncol 2008; 3: 1-5.

7. Sorbe B, Soderstrom K. Treatment of vaginal recurrences in endometrial carcinoma by high-dose-rate brachytherapy. Anticancer Res 2013; 33: 241-248.

8. Vargo J, Kim H, Houser C et al. Definitive salvage for vaginal recurrence of endometrial cancer: The impact of modern intensity-modulated-radiotherapy with image-based HDR brachytherapy and the interplay of the PORTEC 1 risk stratification. Radiother Oncol 2014; 113: 126-131.

9. Jhingran A, Burke TW, Eifel PJ. Definitive radiotherapy for patients with isolated vaginal recurrence of endometrial carcinoma after hysterectomy. Int J Radiat Oncol Biol Phys 2003; 56: 1366-1372.

10. Mock U, Kucera H, Fellner C et al. High-dose-rate (HDR) brachytherapy with or without external beam radiotherapy in the treatment of primary vaginal carcinoma: long-term results and side effects. Int J Radiat Oncol Biol Phys 2003; 56: 950-957.

11. Huh WK, Straughn JM, Mariani A et al. Salvage of isolated vaginal recurrences in women with surgical stage I endometrial cancer: a multiinstitutional experience. Int J Gynecol Cancer 2007; 17: 886-889.

12. Rajagopalan M, Xu K, Lin J et al. Patterns of care and brachytherapy boost utilization for vaginal cancer in the United States. Pract Radiat Oncol 2015; 5: 56-61.

13. Yeh A, Marcus R, Amdur R et al. Patterns of failure in carcinoma of the vagina treated with definitive radiotherapy alone: What is the appropriate field volume? Int J Radiat Oncol Biol Phys 2000; 48: 126-127.

14. Rajagopalan M, Xu K, Sukumvanich P et al. Adoption and impact of concurrent chemoradiation therapy for vaginal cancer: A National Cancer Data Base (NCDB) study. Gynecol Oncol 2014; 135: 495-502.

15. Samant R, Lau B, EC. Primary vaginal cancer treated with concurrent chemoradiation using cis-platinum. Int J Radiat Oncol Biol Phys 2007; 69: 746-750.

16. Miyamoto D, Viswanathan A. Concurrent Chemoradiation for Vaginal Cancer. PLoS ONE 2013; 8: e65048.

17. Li S, Aref I, Walker W et al. Effects of prescription depth, cylinder size, treatment length, tip space, and curved end on doses in high-dose-rate vaginal brachytherapy. Int J Radiat Oncol Biol Phys 2007; 67: 1268-1277.

18. Nout RA, Smit VT, Putter H et al. Vaginal brachytherapy versus pelvic external beam radiotherapy for patients with endometrial cancer of high-intermediate risk (PORTEC-2): an open-label, non-inferiority, randomized trial. Lancet 2010; 375: 816-823.

19. Beriwal S, Demanes DJ, Erickson B et al. American Brachytherapy Society consensus guidelines for interstitial brachytherapy for vaginal cancer. Brachytherapy 2012; 11: 68-75.

20. Shukla P, Chopra S, Engineer R et al. Quality Assurance of Multifractionated Pelvic Interstitial Brachytherapy for Postoperative Recurrences of Cervical Cancers: A Prospective Study. Int J Radiat Oncol Biol Phys 2012; 82: 617-622.

21. Jamema S, Mahantshetty U, Tanderup K et al. Inter-application variation of dose and spatial location of $\mathrm{D}_{2 \mathrm{~cm}}{ }^{3}$ volumes of OARs during MR image based cervix brachytherapy. Radiother Oncol 2013; 107: 58-62. 
22. Demanes DJ, Rege S, Rodriquez RR et al. The use and advantages of a multichannel vaginal cylinder in high-dose rate brachytherapy. Int J Radiat Oncol Biol Phys 1999; 44: 211-219.

23. Kim H, Rajagopalan M, Houser C et al. Dosimetric comparison of multichannel with one single-channel vaginal cylinder for vaginal cancer treatments with high-dose-rate brachytherapy. Brachytherapy 2014; 13: 263-267.

24. Bahadur R, Constantinescu C, Hassouna A et al. Single versus multichannel applicator in high-dose-rate vaginal brachytherapy optimized by inverse treatment planning. J Contemp Brachytherapy 2014; 6: 362-370.

25. Vargo J, Kim H, Houser C, et al. Image-based multichannel vaginal cylinder brachytherapy for vaginal cancer. Brachytherapy 2015; 14: 9-15.

26. Nag S, Gupta N. A simple method of obtaining equivalent doses for use in HDR brachytherapy. Int J Radiat Oncol Biol Phys 2000; 46: 507-513.

27. Patankar S, Tergas A, Deutsch I et al. High versus low-dose rate brachytherapy for cervical cancer. Gynecol Oncol 2015; 136: 534-541.

28. Viswanathan A, Creutzberg C, Craighead P et al. International Brachytherapy Practice Patterns: A Survery of the Gynecologic Cancer Intergroup (GCIC). Int J Radiat Oncol Biol Phys 2012; 82: 250-255.

29. Viswanathan A, Erickson B. Three-Dimensional Imaging in Gynecologic Brachytherapy: A Survey of the American Brachytherapy Society. Int J Radiat Oncol Biol Phys 2010; 76: 104-109.

30. Maruyama Y, Ezzell G, Porter AT et al. Afterloading high dose rate intracavitary vaginal cylinder. Int J Radiat Oncol Biol Phys 1994; 30: 473-476.

31. Kubo H, Glasgow G, Pethel $T$ et al. High dose-rate brachytherapy treatment delivery: Report of the AAPM Radiation Therapy Committee Task Group No. 59. Med Phys 1998; 25: 375-403.

32. Pötter R, Haie-Meder C, Van Limbergen E et al. Recommendations from gynaecological (GYN) GEC ESTRO working group (II): Concepts and terms in 3D image-based treatment planning in cervix cancer brachytherapy - 3D dose volume parameters and aspects of 3D image-based anatomy, radiation physics, radiobiology. Radiother Oncol 2006; 78: 67-77.

33. Georg P, Lang S, Dimopoulos JC et al. Dose-volume histogram parameters and late side effects in magnetic resonance image-guided adaptive cervical cancer brachytherapy. Int J Radiat Oncol Biol Phys 2011; 79: 356-362.

34. Lee L, Viswanathan A. Predictors of Toxicity After Imageguided High-dose-rate Interstitial Brachytherapy for Gynecologic Cancer. Int J Radiat Oncol Biol Phys 2012; 84: 1192-1197.

35. Viswanathan AN, Beriwal S, De Los Santos JF et al. American Brachytherapy Society consensus guidelines for locally advanced carcinoma of the cervix. Part II: High-dose-rate brachytherapy. Brachytherapy 2012; 11: 47-52.

36. Small W, Beriwal S, Demanes DJ et al. American Brachytherapy Society consensus guidelines for adjuvant vaginal cuff brachytherapy after hysterectomy. Brachytherapy 2012; 11: 58-67.

37. Hintz BL, Kagan AR, Chan P et al. Radiation tolerance of the vaginal mucosa. Int J Radiat Oncol Biol Phys 1980; 6: 711-716.

38. Murakami N, Kasamatsu T, Sumi M et al. Vaginal tolerance of CT based image-guided high-dose rate interstitial brachytherapy for gynecological malignancies. Radiat Oncol 2014; 9: 31.

39. Fidarova E, Berger D, Schussler $S$ et al. Dose volume parameter $\mathrm{D}_{2 \mathrm{cc}}$ does not correlate with vaginal side effects in individual patients with cervical cancer treated within a defined treatment protocol with very high brachytherapy doses. Radiother Oncol 2010; 97: 76-79.
40. Rajagopalan M, Kannan N, Kim H et al. Urethral dosimetry and toxicity with high-dose-rate interstitial brachytherapy for vaginal cancer. Brachytherapy 2013; 12: 248-253.

41. Liao Y, Dandekar V, Chu J et al. Reporting small bowel dose in cervix cancer high-dose-rate brachytherapy. Med Dosim 2015; epub ahead of print.

42. Curran W, Whittington R, Peters A et al. Vaginal Recurrences of Endometrial Carcinoma: The Prognostic Value of Staging by a Primary Vaginal Carcinoma System. Int J Radiat Oncol Biol Phys 1988; 15: 803-808.

43. Stock R, Mychalczak B, Armstrong J et al. The Importance of Brachytherapy Technique in the Management of Primary Carcinoma of the Vagina. Int J Radiat Oncol Biol Phys 1992; 24: 747-753.

44. Morgan J, Reddy S, Sarin P et al. Isolated Vaginal Recurrences of Endometrial Carcinoma. Radiology 1993; 189: 609-613.

45. Sears J, Greven K, Hoen H et al. Prognostic Factors and Treatment Outcome for Patients with Locally Recurrent Endometrial Cancer. Cancer 1994; 74: 1303-1308.

46. Chyle V, Zagars G, Wheeler J et al. Definitive Radiotherapy for Carcinoma of the Vagina: Outcome and Prognostic Factors. Int J Radiat Oncol Biol Phys 1996; 35: 891-905.

47. Fine B, Piver S, McAuley M et al. The Curative Potential of Radiation Therapy in the Treatment of Primary Vaginal Carcinoma. Am J Clin Oncol 1996; 19: 39-44.

48. Jereczek-Fossa B, Badzio A, Jassem J. Recurrent Endometrial Cancer After Surgery Alone: Results of Salvage Radiotherapy. Int J Radiat Oncol Biol Phys 2000; 48: 405-413.

49. Wylie J, Irwin C, Pintilie M et al. Results of radical radiotherapy for recurrent endometrial cancer. Gynecol Oncol 2000; 77: 66-72.

50. Lin L, Grigsby P, Powell M et al. Definitive Radiotherapy in the Management of Isolated Vaginal Recurrences of Endometrial Cancer. Int J Radiat Oncol Biol Phys 2005; 63: 500-504.

51. Pai H, Souhami L, Clark B et al. Isolated Vaginal Recurrences of Endometrial Carcinoma: Treatment Results Using HighDose-Rate Intracavitary Brachytherapy and External Beam Radiotherapy. Gynecol Oncol 1997; 66: 300-307.

52. Lieskovsky Y, Demanes D. Combination High-Dose-Rate Brachytherapy and External Beam Radiation Therapy for the Treatment of Primary Vaginal Cancer: 5-Year Results. Int J Radiat Oncol Biol Phys 2004; 60: S308.

53. Dimopoulos J, Schmid M, Fidarova E et al. Treatment of Locally Advanced Vaginal Cancer with Radiochemotherapy and Magnetic Resonance Image-Guided Adaptive Brachytherapy: Dose-Volume Parameters and First Clinical Results. Int J Radiat Oncol Biol Phys 2012; 82: 1880-1888.

54. Beriwal S, Rwigema J, Higgins E et al. Three-dimensional image-based high-dose-rate interstitial brachytherapy for vaginal cancer. Brachytherapy 2012; 11: 176-180.

55. Lee L, Damato A, Viswanathan A. Clinical outcomes following 3D image-guided brachytherapy for vaginal recurrence of endometrial cancer. Gynecol Oncol 2013; 131: 586-592. 